\title{
Perforated Mitral Valve Aneurysm Associated with Libman-Sacks Endocarditis
}

\author{
Tomoyuki Takayama ${ }^{1}$, Masanori Teramura ${ }^{1}$, Hiroshi Sakai ${ }^{1}$, Shinji Tamaki ${ }^{1}$, \\ Tabito Okabayashi ${ }^{1}$, Takeshi Kawashima ${ }^{1}$, Takashi Yamamoto ${ }^{2}$, Minoru Horie ${ }^{2}$, \\ Tomoaki Suzuki ${ }^{3}$ and Tohru Asai ${ }^{3}$
}

\begin{abstract}
The perforation of a mitral valve aneurysm is a rare disease which induces acute mitral regurgitation and is usually induced by infective endocarditis; however, in this case report, acute heart failure was caused by a perforated mitral valve aneurysm that was speculated to be due to Libman-Sacks endocarditis with systemic lupus erythematosis and secondary anti-phospholipid syndrome. Mitral valve plasty was performed and thereafter heart failure improved.
\end{abstract}

Key words: mitral valve aneurysm, anti-phospholipid syndrome, Libman-Sacks endocarditis, mitral valve plasty

(Inter Med 47: 1605-1608, 2008)

(DOI: 10.2169/internalmedicine.47.1068)

\section{Introduction}

The perforation of a mitral valve aneurysm is a rare etiology of acute mitral regurgitation. It has been reported that mitral valve aneurysm is typically induced by infectious endocarditis (1); however, in this case report, the mitral valve aneurysm was speculated to be induced by systemic lupus erythematosis (SLE) and secondary anti-phospholipid syndrome (APS).

\section{Case Report}

A 58-year-old man was admitted to hospital with sudden orthopnea. The physical findings were tachypnea, tachycardia, baldness, leukoderma in his cheek, bilateral rales in the lungs, and pansystolic heart murmur at the apex. His body temperature was $35.7^{\circ} \mathrm{C}$. A 12-lead electrocardiogram showed sinus tachycardia, normal QRS complex and normal ST-T segment. Chest X-ray films showed marked pulmonary congestion, and cardiomegaly of 58\% was observed in the cardiothoracic ratio (Fig. 1). Laboratory tests revealed a white blood cell count of $12,500 / \mu \mathrm{L}$, aspartate aminotransferase $36 \mathrm{IU} / \mathrm{L}$, lactase dehydrogenase $290 \mathrm{IU} / \mathrm{L}$, creatine kinase $83 \mathrm{IU} / \mathrm{L}$ and C-reactive protein $2.59 \mathrm{mg} / \mathrm{dL}$. Plasma B-type natriuretic peptide levels were $385 \mathrm{pg} / \mathrm{mL}$. Although trans-thoracic echocardiography was poor, severe mitral regurgitation was observed. Thereafter, pulmonary congestion improved after diuretics and a vasodilator were administrated. Trans-esophageal echocardiography (TEE) performed 6 days later showed severe mitral valve regurgitation with mitral valve aneurysm rupture in the A2 scallop and moderate aortic regurgitation; the left atrium and ventricle were not large (Fig. 2). Left ventricular angiography revealed MR grade 3 with a mitral valve aneurysm and AR grade 1 (Fig. 3). Under cardiopulmonary bypass, mitral valve valvuloplasty and annuloplasty were performed. During the operation, A2 scallop in the anterior mitral leaflet was found to be markedly enlarged and prolonged. There was a perforation $(3 \times 1 \mathrm{~mm})$ in the middle of the A2 scallop (Fig. 4). The tip of the valves was thickened. On histological examination, there was marked rupture and degeneration of elastic fibers. Neovessel formation and fibrin deposition were induced in the valve. Inflammatory cells were only slightly in-

${ }^{1}$ Division of Cardiology, Department of Medicine, Kohka Public Hospital, Kohka, ${ }^{2}$ Department of Cardiovascular and Respiratory Medicine, Shiga University of Medical Science, Otsu and ${ }^{3}$ Department of Cardiovascular Surgery, Shiga University of Medical Science, Otsu Received for publication March 3, 2008; Accepted for publication June 3, 2008 Correspondence to Dr. Tomoyuki Takayama, totakayama-circ@umin.ac.jp 
filtrated.

Several blood cultures were negative. Results of immunological and auto-antibody examinations were as follows: anti-nuclear antibody titer, 320 (normal reference titer, <40); anti-cardiolipin beta-2 glycoprotein 1 anti-body $14.5 \mathrm{U} / \mathrm{mL}$ (normal reference <3.5 U/mL); CH50 $44 \mathrm{U} / \mathrm{mL}$; anti-Sm antibody, negative; anti-double-strand DNA-IgG antibody, negative. After operation, the symptoms of heart failure improved, but transient hemolytic anemia, renal dysfunction and convulsive seizure were observed. These were improved by resting cure and treatment with anticonvulsant drugs. He had been treated for cryptogenic facial erythema when young, which was not well defined. He often complained of mild althragia. Therefore, from these criteria, he was diagnosed with SLE and secondary APS, and the mitral valve aneurysm was speculated to be caused, not by infective endocarditis, but by Libman-Sacks endocarditis.

\section{Discussion}

Mitral valve aneurysm has frequently been associated with infective endocarditis (1): however, no history of infective endocarditis could be verified in the present case. It was reported that mitral valve aneurysm is also induced by congenital disease (2), Marfan syndrome (3), syphilis (4), and aortitis (5). Although none of these was detected, he was diagnosed with SLE and secondary anti-phospholipid syndrome. The most frequent cardiac manifestations of antiphospolipid syndrome are heart valve abnormalities (vegetation, valve thickening and dysfunction) (6), which are known as Libman-Sacks endocarditis. Recently, it has reported that Libman-Sacks endocarditis is induced by antiphospholipid antibodies (7). Although the mechanism has not been clearly demonstrated, Hojnik et al (7) reviewed that anti-phospholipid antibodies may promote the formation of valve thrombi and may also act by another mechanism, as indicated by the finding of subendothelial deposits of immunoglobulins, including anti-cardiolipin antibodies, and of co-localized complement components in deformed valves from patients with APS. Valvular disease, for the most part, is mild and asymptomatic; only rarely (4-6\%) do antiphospholipid antibody-positive patients develop valve disease severe enough to require surgical treatment (6). However, to our knowledge, it has not reported that mitral aneurysm is induced by Libman-Sacks endocarditis. Although vulvular abnormalities resulting from Libman-Sacks lesions may predispose patients to bacterial endocarditis (8), we did not observe fever, bacteremia or vascular phenomena in this patient. In addition, he did not have a past history of infective endocarditis. On histological examination in this case, inflammatory cells were only slightly infiltrated and we did not detect microorganisms. Resected tissue was culturenegative. Neovessel formation and fibrin deposition were induced and there was degenerative change with fibrosis in this valve. Libman-Sacks valve lesions are microscopically characterized by fibrin deposits at various stages of fibro-

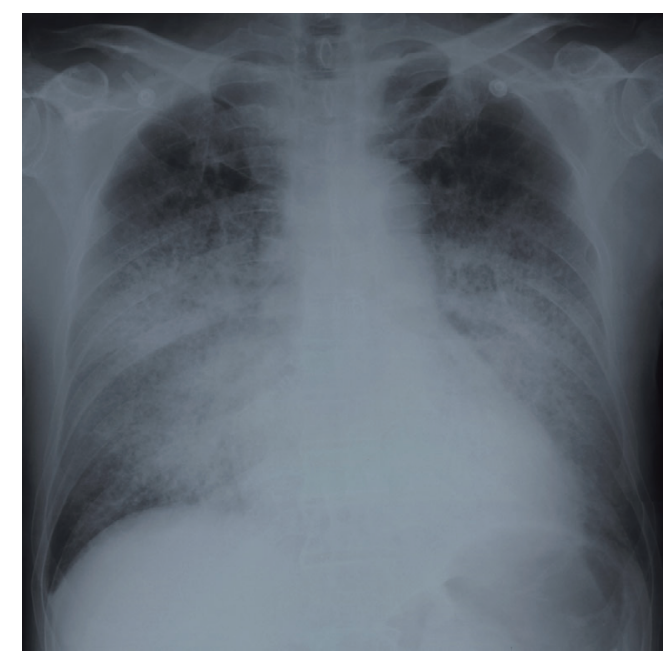

Figure 1. Chest radiography shows cardiomegaly and pulmonary congestion.

blastic organization and neovascularization and by a variable extent of inflammation with mononuclear cell infiltration. The end-stage or healed form of Libman-Sacks verrucous endocarditis is a fibrous plaque (7). Therefore, we speculate that long-term valvular damage by anti-phospholipid antibodies was extensive, and healing may be accompanied by marked scarring, thickening, and deformity of the valve, which most likely leads to severe valve dysfunction. In addition, left ventricular pressure may induce a fragile vulvular site to pathological dilatation, aneurysm and may lead to aneurysm perforation.

In this case, the transthoracic echocardiogram was poor. Although severe mitral valve regurgitation was visualized, mitral valve abnormalities were not vivid, while TEE clearly visualized the perforation of the mitral valve aneurysm. In most cases of valvular disease, it is necessary to perform TEE to obtain good images of valvular lesions.

Mitral valve valvuloplasty and annuloplasty are performed as therapy for the mitral valve aneurysm. APS patients have a high risk of thrombosis. Furthermore, mitral valve replacement in APS patients increases the risk of thrombosis. It was reported that it is important to perform the plasty as far as possible, and to administer effective anticoagulation treatment to prevent complications for patients with SLE and APS (9). Although mitral valve replacement is not performed, lifetime warfarin treatment is thought to be needed. Antithrombotic therapy is certainly indicated as secondary prevention in patients with anti-phospholipid antibodyassociated valvular disease who have already experienced a thromboembolic event (10). As patients with APS are prone to repeated thrombotic episodes, especially in the first few months after withdrawal of oral anticoagulants, long-term, possibly lifelong anticoagulation is required in the presence of persistently elevated anti-phospholipid antibodies titers (11). Although the present patient did not have a history of thrombosis, anti-cardiolipin beta- 2 glycoprotein 1 antibody was persistently elevated; therefore, the risk of thrombosis in the future is thought to be high and continuous warfarin 

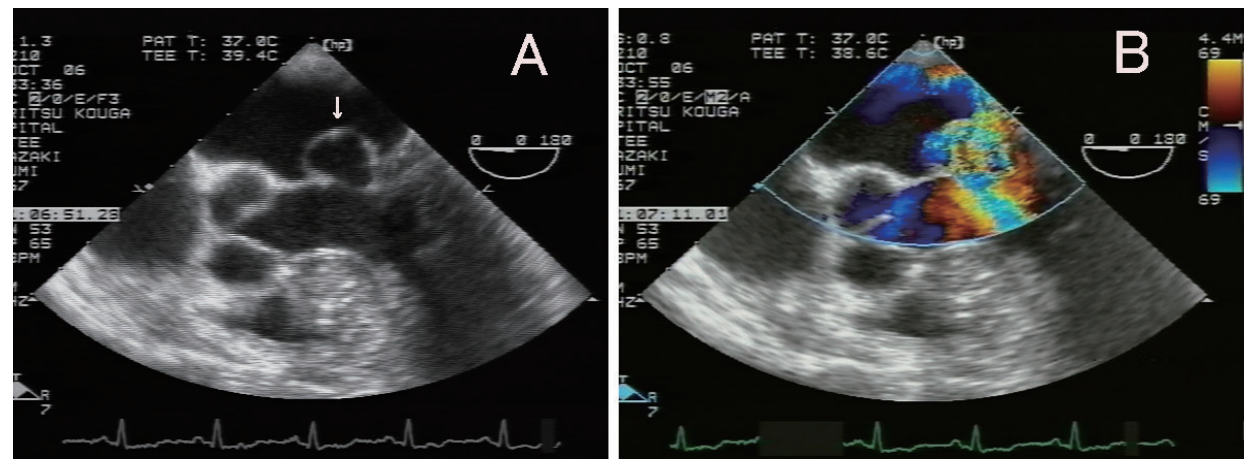

Figure 2. Trans-esophageal echocardiography shows mitral valve aneurysm on the anterior leaflet (Panel A) and severe mitral valve regurgitation with mitral valve aneurysm rupture at systole (Panel B).

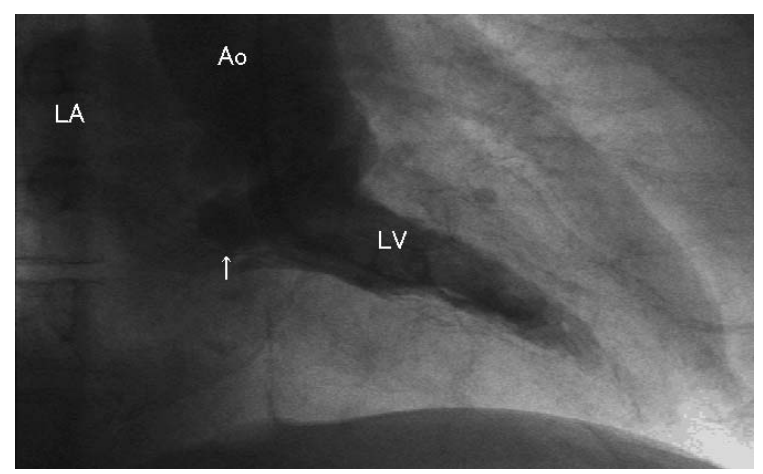

Figure 3. Left ventricular angiography shows severe mitral valve regurgitation with the mitral valve aneurysm.

treatment is indispensable to prevent thrombosis.

There is no direct evidence that treatment with corticosteroids can prevent valvular damage (12). In the present case after operation, SLE was exacerbated temporarily but thereafter, the activity of SLE became stable; therefore, we did not administer corticosteroids. Surgical therapy for asymptomatic valvular aneurysm is controversial as it has a high risk of thrombus formation, infection and perforation,

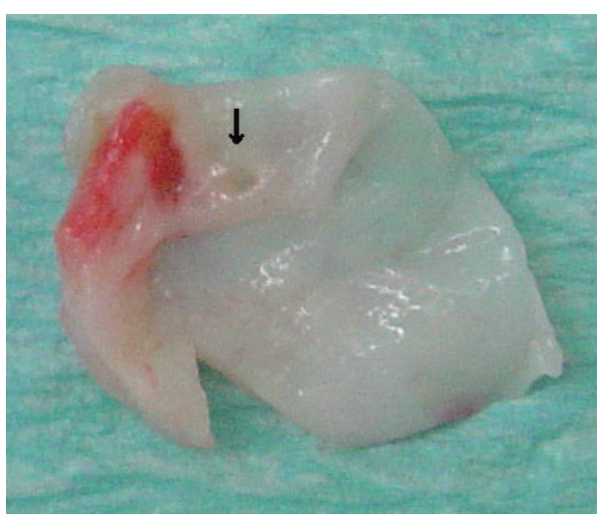

Figure 4. This shows mitral valve aneurysm removed by operation. Arrow line shows pin-hole rupture in the middle of the mitral valve aneurysm.

and needs to be followed up carefully.

In conclusion, we describe a 58-year-old man with perforation of a mitral valve aneurysm, which was speculated to be induced by anti-phosphlipid syndrome. To our knowledge, this such a case has not been described previously.

\section{References}

1. Ichikawa Y, Soma T, Yamazaki I, Isoda S, Kondo J, Matsumoto A. A case of aortic and mitral regurgitation with two aneurysms in the mitral valve. Nippon Kyobu Geka Gakkai Zasshi 42: 13501354, 1994.

2. Musallam SN, McCall M. Aneurysm of the mitral valve. J Okla State Med Assoc 47: 59-63, 1954.

3. Edynak GM, Rawson AJ. Ruptured aneurysm of the mitral valve in a marfan-like syndrome. Am J Cardiol 11: 674-677, 1963.

4. Turner PP, Davies JNP. A large aneurysm of the mitral valve. Am Heart J 66: 538-541, 1963.

5. Kawai S, Oigawa T, Sunayama S, et al. Mitral valve aneurysm as a sequela of infective endocarditis: review of pathologic findings in Japanese cases. J Cardiol 31 Suppl 1: 19-33; discussion 34-36, 1998.

6. Tincani A, Rebaioli CB, Taglietti M, Shoenfeld Y. Heart involvement in systemic lupus erythematosus, anti-phospholipid syn- drome and neonatal lupus. Rheumatology 45(suppl 4): iv8-iv13, 2006.

7. Hojnik M, George J, Ziporen L, Shoenfeld Y. Heart valve involvement (Libman-Sacks Endocarditis) in the antiphospholipid syndrome. Circulation 93: 1579-1587, 1996.

8. Lehman TJA, Palmeri ST, Hastings C, Klippel JH, Plotz PH. Bacterial endocarditis complicating systemic lupus erythematosus. J Rheumatol 10: 655-658, 1983.

9. Yoshida M, Sasako Y, Kobayashi J, Minatoya K, Bando K, Kitamuta S. Mitral valve plasty in systemic lupus erythematosus in the setting of antiphospholipid syndrome. Jpn J Thorac Cardiovasc Surg 48: 391-393, 2000.

10. Khamashta MA, Cuadrado MJ, Mujic F, Taub NA, Hunt BJ, Hughes GRV. The management of thrombosis in the antiphospholipid-antibody syndrome. N Engl J Med 332: 993-997, 1995. 
Inter Med 47: 1605-1608, 2008 DOI: 10.2169/internalmedicine.47.1068

11. Rosove MH, Brewer PMC. Antiphospholipid thrombosis: clinical course after the first thrombotic event in 70 patients. Ann Intern Med 117: 303-308, 1992.

12. Galve E, Candell-Riera J, Pigrau C, Permanyer-Miralda G, Garcia-
Del-Castillo H, Soler-Soler J. Prevalence, morphologic types, and evolution of cardiac valvular disease in systemic lupus erythematosus. N Engl J Med 319: 817-823, 1988.

(C) 2008 The Japanese Society of Internal Medicine http://www.naika.or.jp/imindex.html 